\title{
Comparison of mobility device delivery within Department of Veterans Affairs for individuals with multiple sclerosis versus spinal cord injury
}

\author{
Fabrisia Ambrosio, PhD, MPT; ${ }^{1-3}$ Michael L. Boninger, MD; ${ }^{1-3^{*}}$ Shirley G. Fitzgerald, PhD; ${ }^{1-2}$ Sandra L. \\ Hubbard, PhD; ${ }^{1-2}$ Steven R. Schwid, MD; ${ }^{4-5}$ Rory A. Cooper, PhD ${ }^{1-2}$ \\ ${ }^{1}$ Department of Rehabilitation Sciences and Technology, School of Health and Rehabilitation Sciences, University of \\ Pittsburgh, Pittsburgh, PA; ${ }^{2}$ Human Engineering Research Laboratories, Department of Veterans Affairs Pittsburgh \\ Healthcare System, Pittsburgh, PA; ${ }^{3}$ Department of Physical Medicine and Rehabilitation, School of Medicine, Univer- \\ sity of Pittsburgh, Pittsburgh, PA; ${ }^{4}$ Rochester Multiple Sclerosis Center, Rochester, NY; ${ }^{5}$ Department of Neurology, \\ University of Rochester, Rochester, NY
}

\begin{abstract}
Individuals with multiple sclerosis (MS) report decreased satisfaction with their mobility devices compared with individuals with spinal cord injuries (SCIs). This study (1) investigated the demographic differences between veterans with MS (V-MS) and veterans with SCI (V-SCI) who were issued a wheelchair by the Veterans Health Administration (VHA) and (2) described differences in mobility device prescription. We merged two VHA databases to obtain demographic and wheelchair information for all V-MS and V-SCI in 2000 and 2001. Descriptive information for issued wheelchairs was available for 2,154 V-MS and V-SCI. We found that V-MS were significantly less likely to receive higher quality wheelchairs (manual or power) compared with V-SCI $(p<0.001)$. The disparity in VHA wheelchair prescription between these two groups indicates a need for further research regarding the assistive device prescription process in these populations.
\end{abstract}

Key words: assistive technology, manual wheelchair, multiple sclerosis, power wheelchair, prescription, rehabilitation, scooter, spinal cord injury, veteran, wheelchair.

\section{INTRODUCTION}

Approximately 13.1 million people use assistive devices in the United States for mobility, communication, and assistance with activities of daily living (ADL) [1]. According to the U.S. Assistive Technology Act of 1998, assistive technology (AT) is defined as "any item, piece of equipment, or product system, whether acquired commercially off the shelf, modified, or customized, that is used to increase, maintain, or improve functional capabilities of individuals with disabilities" [2]. While technological advancements have allowed for a sophisticated array of assistive devices, the effectiveness of the technology is only as good as the fit between the user and the assistive device [3]. The match between the person and the technology depends on the specific needs of the individual, his or her functional limitations and disability, and the capability of the technology to meet these needs.

\footnotetext{
Abbreviations: $\mathrm{ADL}=$ activities of daily living, $\mathrm{AT}=$ assistive technology, ICD-9 = International Classification of Diseases, 9th Revision, MS = multiple sclerosis, NPCD = National Patient Care Database, NPPD = National Prosthetics Patient Database, $\mathrm{SCI}=$ spinal cord injury, $\mathrm{SD}=$ standard deviation, VA = Department of Veterans Affairs, VHA = Veterans Health Administration, $\mathrm{V}-\mathrm{MS}=$ veterans with $\mathrm{MS}, \mathrm{V}-\mathrm{SCI}=$ veterans with SCI.

*Address all correspondence to Michael L. Boninger, MD; Human Engineering Research Laboratories, VA Pittsburgh Healthcare System, 7180 Highland Drive, 151R-1, Pittsburgh, PA 15206; 412-365-4850; fax: 412-365-4858.

Email: boninger@pitt.edu

DOI: 10.1682/JRRD.2006.02.0016
} 
This match is important given the correlation between mobility and quality of life [4-5]. With this in mind, researchers have increased their efforts to investigate the process by which AT is issued to consumers and the indicators of quality prescription process. Concern continues to exists, however, that discrepancies in wheelchair prescription based on diagnosis may lead to differences in the appropriateness of the device and AT satisfaction.

One study of 14 men and women with a newly acquired spinal cord injury (SCI) had subjects rate their satisfaction with AT 1 month after discharge from acute rehabilitation [6]. This study found that 64.3 percent of the respondents indicated that they were "satisfied" with their AT and that AT satisfaction, as measured by the Assistive Technology Device Predisposition Assessment, was positively correlated with life satisfaction, as measured by the Satisfaction with Life Scale. Siösteen et al. suggested that people with adequate resources had lives as fulfilling as those of a matched group of nondisabled persons [7]. Along these lines, Cook found that 5 years after SCI, a majority of individuals were functioning comparably to uninjured control subjects in terms of life satisfaction and self-perceived adjustment [8]. Similarly, Cushman and Hassett studied 43 people 10 and 15 years after their SCIs and collected data on the subjects' perceived quality of life [9]. They found that most subjects saw their life as comparable to, or somewhat better than, that of age-matched peers.

In contrast to individuals with SCI, AT satisfaction in persons with multiple sclerosis (MS) is low [10]. Finlayson et al. conducted a study in Canada of individuals with MS who used a mobility device and found that 61 percent used a manual wheelchair and only 8.2 percent used a power wheelchair [11]. This result is disturbing considering Fay et al. found that individuals with MS who use a manual wheelchair generally implement ineffective propulsion patterns and exert excessive forces on the pushrim, thereby increasing the likelihood of fatigue and upper-limb injury [12]. Therefore, Perks et al.'s result that 59 percent of individuals with MS state that their current wheelchair does not meet their mobility needs is not surprising [10]. Finally, Wheeler and colleagues found that when compared with other wheelchair users, individuals with MS are particularly skeptical, critical, and questioning about healthcare providers' explanations regarding mobility device prescription [13].

Clearly, the variability of symptoms in persons with MS leads to dilemmas in clinical decision making related to mobility device prescription. When is a good time to consider a switch to wheeled mobility? What is the best type of wheeled mobility? Are different types of wheeled mobility issued to individuals with MS compared with individuals who have a clearer clinical course, such as those with SCI?

We propose that differences in AT satisfaction in individuals with MS compared with individuals with SCI may be closely linked to differences in the quality of the devices issued. Although these two diagnoses are very different and symptom presentation and progression are likely to vary greatly between the two groups, we can argue that once the decision to transition to a wheeled mobility device is made, all individuals are equally entitled to a wheelchair that will best meet their needs. Successful integration and independent functioning within society depend on the provision of an appropriate wheelchair, with features that are customized to best match the user's needs, preferences, and environment [14-15].

As an initial step in investigating the wheeled mobility prescription process for individuals with MS, we characterized the demographics of veterans with MS (V-MS) and veterans with SCI (V-SCI) who received a wheeled mobility device from the Veterans Health Administration (VHA). We also compared the wheeled mobility devices issued to V-MS and V-SCI. Specifically, we investigated the differences in the types of manual and power wheelchairs and scooters issued to these veterans. Although we had no data available regarding functional limitations of the two groups, we hypothesized, based on clinical observation, that V-MS were more likely to receive low-grade depot-style wheelchairs as their primary means of mobility, which is significant because depot wheelchairs are intended for temporary use only (e.g., during a stay in a rehabilitation hospital) and have been shown to have markedly decreased half-lives when compared with lightweight and ultralightweight wheelchairs [16]. In fact, depot wheelchairs are not likely to survive regular consumer use, making them a poor investment [16]. In addition, an increased physiological demand is required for the propulsion of standard wheelchairs when compared with ultralightweight wheelchairs [17]. This increased demand is significant given the fatigue limitations common in individuals with MS. This study is an initial step in investigating the provision of wheeled mobility devices to V-SCI versus V-MS. Findings are intended to be hypothesis-generating, and we anticipate facilitating a foundation by which future efforts can investigate in 
greater detail the prescription of wheeled mobility devices across diagnoses.

\section{METHODS}

\section{Study Design}

In this study, we used a retrospective design to analyze 2 years of data, 2000 and 2001, from two different VHA databases: the National Patient Care Database (NPCD) and the National Prosthetics Patient Database (NPPD) [18]. The NPPD contains data regarding orthotic, prosthetic, and sensory devices distributed to veterans across the United States, including the first time the device was issued and any repairs or replacements. The NPPD provides valuable information regarding the distribution of AT to veterans with a wide variety of diagnoses. We used the NPCD to obtain demographic information for individuals, including date of birth, sex, race/ ethnicity, and primary diagnosis.

The records from the NPCD and the NPPD were merged to create a data subset with unique identifiers. That is, individuals were included in the system only once, even if they received more than one wheelchair over the 2 years. The NPPD is a database that holds a record of the assistive devices issued to veterans. For this study, we investigated NPPD information on the type of wheelchair issued and the "create date," the date on which the wheelchair was ordered. Prior to combining the data and creating the data subset, we linked the two databases while maintaining patient confidentiality according to the Health Insurance Portability and Accountability Act of 1996 regulations. In linking the data between the two databases, we created a crosswalk file from NPPD to pair the scrambled Social Security numbers with the appropriate match in the NPCD using SAS (SAS Institute Inc, Cary, North Carolina). The resulting data set was further limited to variables that only included V-MS or V-SCI. Data sets were deidentified and patient identification numbers were scrambled.

\section{Description of Data}

Hubbard et al. described the processing of the data from the merged database in detail [19]. Briefly, the number of entries for each variable was calculated as the total number of entries minus missing values for that variable. Age was calculated as the time in years between the create date and the birth date of the individual. All V-MS or V-
SCI were included in the data set. If a veteran had a diagnosis of both SCI and MS, he or she was grouped with V-MS, because MS may be considered a type of SCI. V-SCI were identified if they were issued an International Classification of Diseases, 9th Revision (ICD-9) code indicating a specific diagnosis, with an SCI level, such as "cervical 1-2 spinal cord injury," noted. Individuals with both tetraplegia and paraplegia due to SCI were included in the SCI group.

For type of wheelchair, we created a ranking system of eight wheelchair classes to condense the Medicare K-codes (Healthcare Common Procedure Coding System) [20], a system used to describe the type of wheelchair issued [19]. The new ranking system was based on wheelchair function, weight, and adjustability. This ranking system is described in detail elsewhere [21]. If the same individual received a wheelchair in 2000 and/or 2001, only the wheelchair for the first year was included so the data set comprised only unique entries.

\section{Statistical Analysis}

\section{Demographic Characteristics}

We used descriptive statistics to compare demographic information between the two groups, including the number of entries per diagnosis. We used mean \pm standard deviation (SD) values to describe the age of each group and frequency counts to describe sex and race/ethnicity. All variables were tested for normal distribution. We used an independent samples $t$-test to compare the ages of the two groups.

To increase the power of our analysis, we collapsed the race/ethnicity variable into two general groups, white and minority (Hispanic black, Hispanic white, American Indian, black, Asian, and unknown). We used a chisquare analysis to compare the distribution of race/ ethnicity between the two groups.

\section{Comparison of Devices}

We used frequency counts to describe the number and type of manual wheelchairs, power wheelchairs, and scooters issued to each group over both years.

To investigate whether the types of wheeled mobility devices (manual or power wheelchair or scooter) issued to V-MS and V-SCI were significantly different, we used a chi-square analysis. Groups were considered significantly different at $p<0.001$. This level was selected to indicate significant differences between the comparison groups because the sample size for this study was large. With a 
large sample size, the likelihood of observing statistically significant, but not clinically significant, differences is increased, which we attempted to minimize by using a stricter cutoff point for significance.

\section{RESULTS}

According to our database, a total of 7,076 V-SCI or V-MS received wheelchairs between 2000 and 2001; however, because of missing data, we only evaluated the types of wheelchairs issued to 2,154 veterans. The remaining 4,922 veterans received wheelchairs, but we did not have enough information on their demographics or the types of wheelchairs they received to include them in the analysis. Statistical analyses comparing sex, race/ ethnicity, age, and diagnoses of the individuals with incomplete information versus those with complete information who were included in our analysis revealed no significant differences.

\section{Demographic Characteristics}

Of veterans considered, a total of 791 were V-SCI, 90 of which had tetraplegia due to SCI, and 1,363 were V-MS. According to Hubbard et al., the average age of all veterans who received a wheelchair or scooter in 2000 or 2001 was approximately 67.0 years [19]. In our study, the ages for all V-MS or V-SCI who received wheelchairs in 2000 and 2001 ranged from 20.6 to 100.2 years, with a mean \pm SD of $54.4 \pm 12.8$ (Table 1). An independent samples $t$-test indicated a significant difference $(p<0.001)$ between the ages of the V-SCI and the V-MS. Of all veterans considered, 92.3 percent were male. For each of the two groups, males also represented the majority (Table 1). White veterans made up 67.9 percent of V-SCI and 85.0 percent of V-MS. We found a significant difference $(p<0.001)$ in racial/ethnic distribution between the two diagnoses.

\section{Comparison of Devices}

V-SCI received approximately the same number of manual wheelchairs as power wheelchairs $(49.8 \%$ and 43.7\%, respectively) (Table 2). Scooters were the least frequently prescribed mobility device at only 6.4 percent of all devices issued to V-SCI (Table 2). For V-MS, manual wheelchairs were the most commonly issued mobility device (44.7\%), followed by power wheelchairs (33.7\%), and finally scooters (21.6\%). A chi-square analysis revealed a significant difference $(p<0.001)$ between manual wheelchairs, power wheelchairs, and scooters issued to veterans according to diagnosis.

When comparing the type of manual wheelchair issued, we found that ultralightweight wheelchairs were the most common type of manual wheelchair issued to V-SCI (Table 3). For this same group, a depot wheelchair was the least frequently prescribed type of manual wheelchair. On

Table 1.

Age, sex, and race/ethnicity of veterans who received wheeled mobility in 2000 and 2001 for two comparison groups, spinal cord injury (SCI) $(n=791)$ vs multiple sclerosis (MS) $(n=1,363)$.

\begin{tabular}{lcccc}
\hline \multicolumn{1}{c}{ Variable } & SCI & MS & \multicolumn{2}{c}{ Test Statistic* $^{*}$} \\
\cline { 3 - 5 } Age (mean \pm SD) & $52.8 \pm 14.0$ & $55.3 \pm 12.0$ & -4.32 & - \\
Sex (\% male) & 98.0 & 88.9 & - & 57.39 \\
Race/Ethnicity (\% white) & 67.9 & 85.0 & - & 123.41 \\
\hline${ }^{*} p<0.001$ for all comparisons. & & & & \\
SD $=$ standard deviation. & & & \\
\hline \hline
\end{tabular}

Table 2.

Comparison of distribution, $n(\%)$, of manual wheelchairs, power wheelchairs, and scooters issued to veterans with spinal cord injury (SCI) $(n=791)$ vs multiple sclerosis (MS) $(n=1,363)$ in 2000 and 2001. Percentages given as percent of total number of devices issued for each diagnosis.

\begin{tabular}{lccrr}
\hline \multicolumn{1}{c}{ Type } & SCI & MS & Total & $\chi^{2^{*}}$ \\
\hline Manual Wheelchair & $394(49.8)$ & $609(44.7)$ & $1,003(46.6)$ & 102.84 \\
Power Wheelchair & $346(43.7)$ & $460(33.7)$ & $806(37.4)$ & 26.25 \\
Scooter & $51(6.4)$ & $294(21.6)$ & $345(16.0)$ & 171.16 \\
\hline${ }^{*} p<0.001$ for all comparisons. & & & & \\
\hline
\end{tabular}


Table 3.

Comparison of distribution, $n$ (\%), of 3 types of manual wheelchairs, 3 types of power wheelchairs, and scooters issued to veterans with spinal cord injury (SCI) $(n=791)$ vs multiple sclerosis (MS) $(n=$ $1,363)$ in 2000 and 2001. Percentages given as percent of total number of devices issued for each diagnosis.

\begin{tabular}{lrr}
\hline \multicolumn{1}{c}{ Type } & SCI & \multicolumn{1}{c}{ MS } \\
\hline Manual Wheelchair & $101(25.6)$ & $239(39.2)$ \\
Depot & $126(32.0)$ & $285(46.8)$ \\
Lightweight & $167(42.4)$ & $85(14.0)$ \\
Ultralightweight & & \\
Power Wheelchair & $126(31.7)$ & $189(25.1)$ \\
Nonprogrammable & $76(19.1)$ & $154(20.4)$ \\
Miscellaneous & $144(36.3)$ & $117(15.5)$ \\
Custom & $51(12.8)$ & $294(39.0)$ \\
Scooter &
\end{tabular}

the other hand, ultralightweight wheelchairs were the least frequently prescribed manual wheelchairs for V-MS, representing only 14.0 percent of all manual wheelchairs issued. V-MS were more likely to receive a lightweight wheelchair or a depot wheelchair. Specifically, 39.2 percent of V-MS who received manual wheelchairs were issued depot chairs.

For the V-MS, scooters were the most common type of powered mobility device prescribed (Table 3). For the V-SCI, however, a custom power wheelchair was the most common type of powered mobility issued (36.3\%), while only 15.5 percent of the V-MS were issued a custom power wheelchair.

\section{DISCUSSION}

To the best of our knowledge, this is the first study to compare AT prescription between V-MS and V-SCI. For individuals with mobility impairments, AT can greatly affect their ability to participate in ADL and ultimately quality of life. Differences in AT prescription should reflect differences in the functional needs of individuals with SCI versus MS.

We found no significant differences between the demographic characteristics of the group who were eliminated from the analysis and those included in the analysis, which suggests no selection bias based on data entry for the domains we evaluated was present. We did not, however, consider differences in the geographic distribution of individuals with missing data versus those that were included in the analysis. While outside the scope of this article, geographic distribution is a potential cause of selection bias. According to our study, the average age of
V-MS and V-SCI was statistically significantly different. However, because the difference in the mean ages was not more than 3 years, it does not appear to be clinically significant. Furthermore, we found no clinically significant difference in the mean ages of our samples and that of the general population of veterans receiving wheelchairs, which is approximately 55 to 56 years old [19].

Our sex distribution was consistent with the overall distribution of U.S. veterans, since the majority (95.2\%) is male. Similarly, according to Hubbard et al., 96 percent of the veterans who received wheelchairs and scooters between 1999 and 2001 were male [19]. Among the general population, the ratio of males to females with SCI is 4:1 (http://www.emedicine.com/pmr/topic182.htm). However, female individuals with MS outnumber male individuals with MS by $2.6: 1.0$ in the general population (http://www.themcfox.com/multiple-sclerosis/ms-facts/ multiple-sclerosis-facts.htm). This disparity may help explain why males with MS made up approximately 10 percent less of the population compared with males with SCI.

When comparing the types of wheelchairs issued to VMS and V-SCI, we performed chi-square analyses that revealed a significant difference in the general types of wheeled mobility (manual wheelchairs and powered mobility devices) issued ( $\left.\chi^{2}=87.65, p<0.001\right)$. While an approximately equal number of manual and power wheelchairs were issued to V-SCI, manual wheelchairs were the most common category of wheeled mobility issued to V-MS. A recent study conducted in our laboratory investigated the efficacy of manual wheelchair propulsion in individuals with MS $(n=15)$ who use a manual wheelchair as their primary means of mobility [12]. This study found that individuals with MS were unable to maintain a functional speed of wheelchair propulsion when compared with control subjects. Kinetic analysis revealed that with each propulsive stroke of the wheelchair, individuals with MS imparted a force in the opposite direction of forward propulsion, essentially working against themselves every time they pushed the chair. This force would lead to increased energy expenditure during wheelchair propulsion, which is especially significant in this population for whom fatigue is a major limiting factor [22]. Many individuals with MS may be being issued manual wheelchairs, even though such devices are unlikely to be an energy effective means of mobility for them. The prescription of a pushrim-activated power-assisted manual wheelchair or a power wheelchair, for example, may offer the advantage of decreased metabolic demand and, therefore, minimize 
the energy expenditure associated with manual wheelchair propulsion.

Symptom presentation and, consequently, mobility limitation are extremely varied among different individuals with MS. Nonetheless, when the decision to switch to wheeled mobility is made, the individual should be issued the AT device that best addresses his or her physical and functional limitations, such as fatigue levels and neurological impairments, as well as environmental challenges. We may be tempted to view wheelchair prescription for individuals with MS as a supplemental means of mobility for people who retain the ability to walk but have limited endurance. However, issuing a mobility device in which the individual is unlikely to be independent is counterproductive; the rationale behind AT delivery is maximizing the functional abilities of the individual, regardless of diagnosis. In fact, a pilot study conducted by Devitt et al. showed that individuals with MS who used their chair several days a week for only part of the day overwhelmingly reported being "not satisfied" with their wheelchair [23]. Similarly, wheelchair satisfaction was shown to decrease as the assistance needed to propel the wheelchair increased [23]. While the present study is limited by the fact that we have no data on the disease progression and functional abilities of the veterans, it does provide insight as to the types of devices commonly issued to V-MS compared with V-SCI and highlights the need for continued studies that investigate the factors involved in the discrepancies in AT prescription between these two groups.

When we considered the individual types of manual wheelchairs, V-SCI were most likely to receive an ultralightweight wheelchair. On the other hand, only 14 percent of V-MS who were issued a manual wheelchair received an ultralightweight chair. Research has shown that ultralightweight wheelchairs are the best quality of wheelchair in terms of cost-effectiveness and decreased strain on the user's upper limb. Specifically, although ultralightweight wheelchairs are the most expensive type of manual wheelchairs, they have been shown to have a higher number of wheelchair propulsion cycles per dollar than either depot or lightweight chairs [16] and to last 13.2 times longer than depot wheelchairs [24]. These ultralightweight wheelchairs also offer the adjustability needed to minimize the risk for upper-limb injury in individuals who rely on a manual wheelchair as their primary means of mobility. Therefore, the potential dichotomy in the types of manual wheelchairs issued to V-SCI and V-MS is noteworthy.
Our results revealed that V-MS are also less likely than V-SCI to receive a better quality power wheelchair. While V-SCI were most likely to receive a custom power wheelchair, V-MS were most likely to receive a scooter. The increased delivery of scooters to V-MS may be attributed to their ability to perform a stand-pivot transfer, which is unlike many V-SCI. We can also argue that scooters are perceived as less of a symbol of disability when compared with standard power wheelchairs. Because of the unpredictable course of MS, V-MS may find accepting their increased reliance on AT for daily mobility more difficult. They may view the transition as a sign of "giving up." A scooter may offer a means of alleviating fatigue symptoms resulting from ambulation, without the self-perception of increased disability. However, scooters are more difficult to operate indoors than power wheelchairs and may not be effective in the home. A future study should consider if and when V-MS transition to power wheelchairs.

This study is also limited because it does not control for disability severity, service connection, or disease progression/duration. Information regarding the reason for which the mobility device was first issued was not available from the databases analyzed. Clearly, individuals who intend to use their wheeled mobility device parttime are more likely to receive a lower quality wheelchair compared with individuals who will be using the wheelchair as their primary means of mobility.

Another limitation of the study was that within the general population 52 percent of individuals with SCI have paraplegia and 47 percent have tetraplegia (http:// www.sci-info-pages.com/facts.html) and once all the missing data were filtered from the NPPD database for 2000 and 2001, only 11.4 percent of the V-SCI had tetraplegia, indicating that we likely did not capture all veterans with tetraplegia. Clinicians may have coded veterans with tetraplegia due to SCI as another primary diagnosis besides definitive SCI, such as "quadriplegia or quadriparesis." This diagnosis was not included in our analysis because the tetraplegia may have resulted from another condition besides traumatic SCI, such as MS. Similarly, tetraplegia due to SCI is likely accompanied by other secondary diagnoses, such as respiratory problems, which may have been the primary diagnosis of the clinician entering the patient information. Therefore, some discrepancies in the classification of SCI may exist when clinicians input the primary diagnosis into the database. The underrepresentation of veterans with tetraplegia due to SCI likely caused an 
underestimate of the number of power wheelchairs issued to V-SCI. This underestimation would further widen the gap between the general types of wheeled mobility devices (manual wheelchair, power wheelchair, or scooter) issued to veterans with the two diagnoses. This study reveals that future reliability studies should be conducted in ICD-9 coding as it relates to wheelchair prescription within the Department of Veterans Affairs (VA).

These results are specific to V-SCI and V-MS and because of differences in funding policies and patient demographics may not be generalizable to the SCI and MS populations at large. First, while the average age of veterans in the sample we investigated was 55 years, the average age of an individual in the community with an SCI is 31 years; the average age of an individual in the community with MS is 37 years. This difference represents the aging veteran population within the VA. In addition, the type of insurance available to veterans is different than that available to the general population. A previous study has shown that individuals who have public insurance, such as Medicare and Medicaid, receive a lower quality wheelchair compared with individuals who have private insurance [25]. Veterans likely receive a higher overall quality wheelchair than individuals with public insurance because of the increased purchase flexibility of the VA. This difference is not present when the type of wheelchairs issued to individuals from the VA or a Model SCI System are compared. According to Hunt et al., within a Model SCI System, 97 percent of all the manual wheelchairs issued were ultralightweight [25]. Therefore, specialty of the prescriber likely plays a role in the type of wheelchair issued.

V-MS may be issued a poorer quality (heavier and less adjustable) manual wheelchair when compared with V-SCI because clinicians anticipate the progression of this dynamic disease. Clinicians may view the use of a manual wheelchair as an intermediate step in the disease progression of MS to be followed by an increased reliance on a power device for mobility. Since many individuals obtain prescriptions for a manual wheelchair to alleviate some of their daily fatigue, they may not use the wheelchair as their primary means of mobility and are therefore issued a lower quality wheelchair. Ironically, this lower quality wheelchair may lead to an increase in the fatigue symptoms they are attempting to overcome because depot and lightweight chairs are heavier than ultralightweight chairs and, hence, require increased effort and energy expenditure.
Finally, given the acute nature of the onset of SCI, individuals are more likely to receive a prescription for their new mobility device through a team at an inpatient rehabilitation facility. Within Model SCI Systems, for example, the standard of care for individuals with a traumatic SCI is issuance of a customizable manual or power wheelchair [25]. On the other hand, MS is a chronic progressive disease, and symptoms often have a slow onset. With this slow progression in mind, individuals may not be referred to or have the same access to AT specialists for their wheelchair prescription, which may compromise the type of wheelchair being issued. Hoenig et al. conducted a study comparing wheelchair use when prescribed by expert clinicians versus clinicians who had no special training in wheelchair provision [26]. They found that when the wheelchair was prescribed by an expert therapist, individuals used their wheelchair more often. Ultimately, however, the final decision is not solely determined by the professional issuing the device but also by consumer preference. The information obtained from the VHA databases represents the final delivery decision and does not necessarily reflect the recommendations of the clinician alone, which highlights the importance of patient education as to the costs and benefits of each of the available wheeled mobility devices.

\section{CONCLUSIONS}

Our study reveals that a disparity may exist in the types of wheelchairs distributed to V-SCI and V-MS. V-MS may be less likely to receive ultralightweight wheelchairs when they are issued a manual wheelchair, and when powered mobility is considered, they may be less likely to receive programmable customizable power wheelchairs. Instead, V-MS are most likely to receive a scooter.

Although this study is primarily limited by the lack of information regarding disease progression information and satisfaction with mobility device, it does suggest a need for further investigation into the quality and type of wheeled mobility devices issued to V-MS. Specifically, effective solutions for optimizing the provision of AT devices among V-MS should be further investigated and correlated with AT satisfaction. Future studies should also seek to identify standards of care for issuing mobility devices to this population of individuals with MS who have a complicated and unpredictable clinical course. 


\section{ACKNOWLEDGMENTS}

Dr. Hubbard is now with the Rehabilitation Outcomes Research Center, North Florida/South Georgia VA Health System, Gainesville, Florida.

This material was based on work supported by the National Science Foundation's Integrative Graduate Education Research Traineeship Program (grant DGE0333420) and the VA Rehabilitation Research and Development Center of Excellence on Wheelchairs and Associated Rehabilitation Engineering (grant B3142C).

The authors have declared that no competing interests exist.

\section{REFERENCES}

1. LaPlante MP, Carlson D, National Institute on Disability and Rehabilitation Research. Disability in the United States: Prevalence and causes, 1992. Washington (DC): U.S. Department of Education, Office of Special Education and Rehabilitative Services; 1996.

2. Committee on Labor and Human Resources. Assistive Technology Act of 1998: Report. Washington (DC): U.S. Government Printing Office; 1998.

3. Scherer MJ. The Matching Person and Technology (MPT) model and assessment instruments. Revised edition. Webster (NY): The Institute for Matching Person \& Technology; 1998.

4. Aronson KJ. Quality of life among persons with multiple sclerosis and their caregivers. Neurology. 1997;48(1):74-80. [PMID: 9008497]

5. Evers KJ, Karnilowicz W. Patient attitude as a function of disease state in multiple sclerosis. Soc Sci Med. 1996;43(8): 1245-51. [PMID: 8903129]

6. Scherer MJ, Cushman LA. Predicting satisfaction with assistive technology for a sample of adults with new spinal cord injuries. Psych Rep. 2000;87(3 Pt 1):981-87. [PMID: 11191417]

7. Siösteen A, Lundqvist C, Blomstrand C, Sullivan L, Sullivan $\mathrm{M}$. The quality of life of three functional spinal cord injury subgroups in a Swedish community. Paraplegia. 1990;28(8):476-88. [PMID: 2263404$]$

8. Cook DW. A multivariate analysis of motivational attributes among spinal cord injured rehabilitation clients. Int J Rehabil Res. 1981;4(1):5-15. [PMID: 7216573]

9. Cushman LA, Hassett J. Spinal cord injury: 10 and 15 years after. Paraplegia. 1992;30(10):690-96. [PMID: 1448296]

10. Perks BA, Mackintosh R, Stewart CP, Bardsley GI. A survey of marginal wheelchair users. J Rehabil Res Dev. 1994; 31(4):297-302. [PMID: 7869277]

11. Finlayson M, Guglielmello L, Liefer K. Describing and predicting the possession of assistive devices among persons with multiple sclerosis. Am J Occup Ther. 2001; 55(5):545-51. [PMID: 14601815]

12. Fay BT, Boninger ML, Fitzgerald SG, Souza AL, Cooper RA, Koontz AM. Manual wheelchair pushrim dynamics in people with multiple sclerosis. Arch Phys Med Rehabil. 2004;85(6):935-42. [PMID: 15179647]

13. Wheeler G, Krausher K, Cumming C, Jung V, Steadward R, Cumming D. Personal styles and ways of coping in individuals who use wheelchairs. Spinal Cord. 1996;34(6): 351-57. [PMID: 8963989]

14. Scherer MJ, Cushman LA. Measuring subjective quality of life following spinal cord injury: A validation study of the assistive technology device predisposition assessment. Disabil Rehabil. 2001;23(9):387-93. [PMID: 11394589]

15. National Council on Disability [homepage on the Internet]. Washington (DC): National Council on Disability; c2007 [updated 2007 Jul 13; cited 2006 Aug 18]. Study on the financing of assistive technology devices and services for individuals with disabilities: A report to the President and Congress of the United States. March 4, 1993; [about 88 screens]. Available from: http://www.ncd.gov/newsroom/ publications/1993/assistive.htm

16. Fitzgerald SG, Cooper RA, Boninger ML, Rentschler AJ. Comparison of fatigue life for 3 types of manual wheelchairs. Arch Phys Med Rehabil. 2001;82(10):1484-88. [PMID: 11588758]

17. Beekman CE, Miller-Porter L, Schoneberger M. Energy cost of propulsion in standard and ultralight wheelchairs in people with spinal cord injuries. Phys Ther. 1999;79(2): 146-58. [PMID: 10029055]

18. Downs F Jr. The National Prosthetic Patient Database: A national information resource for prosthetic prescriptions written in the VA. J Rehabil Res Dev. 2000;37(3):vii-xi. [PMID: 10917255]

19. Hubbard SL, Fitzgerald SG, Reker DM, Boninger ML, Cooper RA, Kazis LE. Demographic characteristics of veterans who received wheelchairs and scooters from Veterans Health Administration. J Rehabil Res Dev. 2006;43(7): 831-44. [PMID: 17436170$]$

20. Centers for Medicare \& Medicaid Services [homepage on the Internet]. Baltimore (MD): Centers for Medicare \& Medicaid Services; c2007 [updated 2007 May 18; cited 2006 Aug 18]. New information regarding Medicare payment and coding for drugs and biologics; [about 1 screen]. Available from: http://www.cms.hhs.gov/MedHCPCSGenInfo/

21. Hubbard SL, Fitzgerald SG, Vogel B, Reker DM, Cooper RA, Boninger ML. Distribution and cost of wheelchairs and scooters from Veterans Health Administration. J Rehabil Res Dev. 2007;44(4):581-92.

22. Krupp LB, Alvarez LA, LaRocca NG, Scheinberg LC. Fatigue in multiple sclerosis. Arch Neurol. 1988;45(4): 435-37. [PMID: 3355400] 
23. Devitt R, Chau B, Jutai JW. The effect of wheelchair use on the quality of life of persons with multiple sclerosis. Occup Ther Health Care. 2003;17(3/4):63-80.

24. Cooper RA, Robertson RN, Lawrence B, Heil T, Albright SJ, VanSickle DP, Gonzalez J. Life-cycle analysis of depot versus rehabilitation manual wheelchairs. J Rehabil Res Dev. 1996;33(1):45-55. [PMID: 8868417]

25. Hunt PC, Boninger ML, Cooper RA, Zafonte RD, Fitzgerald SG, Schmeler MR. Demographic and socioeconomic factors associated with disparity in wheelchair customizability among people with traumatic spinal cord injury.
Arch Phys Med Rehabil. 2004;85(11):1859-64.

[PMID: 15520982]

26. Hoenig H, Landerman LR, Shipp KM, Pieper C, Pieper C, Richardson M, Pahel N, George L. A clinical trial of a rehabilitation expert clinician versus usual care for providing manual wheelchairs. J Am Geriatr Soc. 2005;53(10): 1712-20. [PMID: 16181170]

Submitted for publication February 17, 2006. Accepted in revised form June 4, 2007. 